\title{
MicroRNA-128a, BMI1 polycomb ring finger oncogene, and reactive oxygen species inhibit the growth of U-87 MG glioblastoma cells following exposure to $\mathrm{X}$-ray radiation
}

\author{
LAN YE ${ }^{1}$, GUANYING YU ${ }^{2}$, CUIHONG WANG ${ }^{1}$, BIN DU $^{2}$, DIANSHUI SUN ${ }^{1}$, JUNLI LIU ${ }^{1}$, \\ TONGGANG QI ${ }^{1}$, XIAOMING YU ${ }^{1}$, WEI WEI ${ }^{1}$, JIAN CHENG ${ }^{1}$ and YUHUA JIANG ${ }^{1}$ \\ ${ }^{1}$ Cancer Center, The Second Hospital of Shandong University, Jinan, Shandong 250033; \\ ${ }^{2}$ Department of Surgery, Jinan Central Hospital, Jinan, Shandong 250014, P.R. China
}

Received October 11, 2014; Accepted July 17, 2015

DOI: $10.3892 / \mathrm{mmr} .2015 .4175$

\begin{abstract}
Radiotherapy is an important therapeutic strategy for the treatment of numerous types of malignant tumors, including glioma. However, radioresistance and anti-apoptotic mechanisms decrease the efficacy of radiotherapy in many patients with glioma. BMI1 polycomb ring finger oncogene (Bmi-1) is an oncogene associated with radioresistance in tumor cells. MicroRNA (miRNA)-128a is a brain-specific miRNA, which suppresses Bmi-1 expression. The present study investigated the effects of various radiation intensities on U-87 MG glioma cells, as well as the role of reactive oxygen species (ROS), Bmi-1, and miRNA-128a in the cellular response to radiotherapy. The response of U-87 MG cells following exposure to $\mathrm{X}$-ray radiation was assessed using a cell growth curve and inhibition ratio. Cell cycle distribution and the levels of intracellular ROS were evaluated by flow cytometry. The mRNA expression levels of Bmi-1 and those of miRNA-128a in U-87 MG cells exposed to X-ray radiation were evaluated by reverse transcription-quantitative polymerase chain reaction. X-ray radiation did not decrease the number of U-87 MG cells; however, it did inhibit cellular growth in a dose-dependent manner. Following exposure to $\mathrm{X}$-ray radiation for $24 \mathrm{~h}$, cell cycle distribution was altered, with an increase in the number of cells in $G_{0} / G_{1}$ phase. The mRNA expression levels of Bmi-1 were downregulated in the 1 and 2 Gy groups, and upregulated in the 6 and 8 Gy groups. The expression levels of miRNA-128a were upregulated in the 1 and 2 Gy groups, and downregulated in the 8 Gy group. The levels of ROS were increased following exposure to $\geq 2 \mathrm{~Gy}$, and treatment with $\mathrm{N}$-acetyl cysteine was able to
\end{abstract}

Correspondence to: Professor Yuhua Jiang, Cancer Center, The Second Hospital of Shandong University, 247 Beiyuan Street, Jinan, Shandong 250033, P.R. China

E-mail: jiangyuhua@sdu.edu.cn

Key words: glioma, microRNA-128a, BMI1 polycomb ring finger oncogene, reactive oxygen species, radioresistance induce radioresistance. These results suggested that U-87 MG cells exhibited radioresistance. High doses of X-ray radiation increased the expression levels of Bmi-1, which may be associated with the evasion of cellular senescence. miRNA-128a and its downstream target gene Bmi-1 may have an important role in the radioresistance of U-87 MG glioma cells. In addition, ROS may be involved in the mechanisms underlying the inhibitory effects of X-ray radiation in U-87 MG cells, and the downregulation of ROS may induce radioresistance.

\section{Introduction}

Glioblastoma is the most aggressive type of malignant brain tumor (1). Therapeutic outcomes have improved with the development of comprehensive treatments, including surgery, radiation and chemotherapy; however, patients with glioblastoma grade IV have a poor long-term prognosis, with an overall survival rate of 1 year $(2,3)$. Recent studies have extensively investigated the cellular and genetic mechanisms underlying glioblastoma progression $(4,5)$. Although the molecular mechanisms underlying glioma radioresistance have yet to be elucidated, they are thought to involve dysregulated gene expression (6).

MicroRNAs (miRNAs) are 19-23 nucleotide RNA molecules, which bind the 3'-untranslated region (3'-UTR) of genes, and regulate gene expression by inhibiting protein translation (7). A previous study demonstrated that miRNAs exert post-transcriptional gene silencing effects (8). In addition, miRNAs mediate the degradation of RNA transcripts (9). Due to the important role of miRNAs in the development, invasion, and metastasis of malignant tumors, previous studies have focused on the role of miRNAs in tumors $(10,11)$. Numerous miRNAs are overexpressed in malignant tumors, as compared with normal tissues (12), and the dysregulation of miRNAs may result in increased tumorigenesis (13).

miRNAs have a significant role in glioma. miRNA-21 has been shown to be overexpressed in glioblastoma, and following the suppression of miRNA-21 expression, glioblastoma growth was inhibited (14). miRNA-184 regulates cell cycle-associated proteins, thereby regulating the proliferation 
of glioma cells (15). Furthermore, miRNA-23a is upregulated in glioma and promotes the invasion of glioma cells (16).

Our previous study investigated the role of BMI1 polycomb ring finger oncogene (Bmi-1) in the response of U-87 MG cells to radiation exposure (17). The results indicated that miRNA-128a was able to bind to the 3'-UTR of Bmi-1 in order to suppress its expression. miRNA-128a is a brain-specific miRNA, which is significantly downregulated in glioma (18). It has previously been demonstrated that, as compared with normal adjacent brain tissue, the expression levels of miRNA-128a in glioblastoma tissue are decreased. The role of miRNA-128a may be associated with the proliferation and self-renewal of glioma stem cells (19). Although the role of miRNA-128a in glioma is well understood, its association with radioresistance in glioblastoma has yet to be elucidated. The present study aimed to investigate the role of miRNA-128a in the growth inhibition of U-87 MG cells exposed to X-ray radiation. The present study hypothesized that U-87 MG cell radioresistance results from suppression of the Bmi-1 oncogene by miRNA-128a.

\section{Materials and methods}

Cell culture. The U-87 MG glioblastoma cell line was obtained from the Cell Bank of Type Culture Collection of Chinese Academy Sciences (Shanghai, China). The U-87 MG cells were cultured in Minimum Essential Medium (MEM; GE Healthcare Life Sciences, Logan, UT, USA) supplemented with $10 \%$ fetal bovine serum (FBS; GE Healthcare Life Sciences) according to the manufacturer's instructions, and incubated in a humidified atmosphere containing $5 \% \mathrm{CO}_{2}$ at $37^{\circ} \mathrm{C}$.

Radiation. The U-87 MG cells were exposed to X-ray radiation using a linear accelerator source (Elekta Synergy ${ }^{\circledR}$ Platform; Elekta, Stockholm, Sweden) at a dose rate of $300 \mathrm{~Gy} / \mathrm{min}$. Prior to radiation, a radiation plan was designed, and the accuracy of the X-ray radiation doses were verified by a radiation therapy physicist using I'mRT MatriXX 2D-ion chamber array (IBA Dosimetry, Schwarzenbruck, Germany).

To investigate the effect of radiation, the cells were exposed on day 0 to various doses of X-ray radiation, including $0 \mathrm{~Gy}$ (control group) and 1, 2, 4, 6 and 8 Gy. All groups of cells were continuously incubated following radiation until the experiments were finished.

Following equivalent doses of radiation, different modes of fractionation may have different effects on tumor cells. To determine the effects of different modes of fractionation, three radiation groups with equivalent radiation doses were assessed in the present study, and compared with the control group, which was administered a 0 Gy dose: The conventional fractionation group was exposed to a $2 \mathrm{~Gy} \mathrm{X}$-ray dose daily from day 0 to day 4 ; the hypofractionation group was exposed to an 8 Gy X-ray dose on day 0 ; the hyperfractionation group was exposed at $1.1 \mathrm{~Gy} \mathrm{X}$-ray dose twice a day from day 0 to day 4 , and the interval between the two treatments was $>6 \mathrm{~h}$.

Cell growth curve and inhibition ratio. The U-87 MG glioblastoma cells were harvested during the logarithmic growth phase and seeded into $24-$ well dishes at a density of $5 \times 10^{4} /$ well in a final volume of $500 \mu \mathrm{l} /$ well. The cells were subsequently exposed to various doses or fractions of X-ray radiation. Every $24 \mathrm{~h}$, the number of cells in three random wells was counted using a cell counter (Inno-Alliance Biotech, Inc., Wilmington, $\mathrm{NC}$, USA), and the mean number of cells were calculated for each time point. A cell growth curve was generated with cell number on the $\mathrm{x}$-axis and the various time points on the $\mathrm{y}$-axis. The inhibition ratio was calculated from the number of radiation group cells divided by the number of control group cells. The results were presented as the mean \pm standard error of three independent experiments.

Cell cycle analysis. To detect the cell cycle phase of the cells following $24 \mathrm{~h}$ exposure to X-ray radiation, the U-87 MG cells were fixed with $70 \%$ ethanol for $30 \mathrm{~min}$. Each sample of the U-87 MG cells was re-suspended in $1 \mathrm{ml}$ phosphate-buffered saline (PBS) supplemented with $1 \%$ FBS, and treated with $500 \mu 1$ PBS containing with $100 \mu \mathrm{g} / \mathrm{ml}$ ribonuclease (Beyotime Institute of Biotechnology, Haimen, China) and $50 \mu \mathrm{g} / \mathrm{ml}$ propidium iodide (Sigma-Aldrich, St. Louis, MO, USA). The samples were subsequently analyzed by flow cytometry (FCM) using the LSR Fortessa Cell Analyzer (BD Biosciences, Franklin Lakes, NJ, USA). A cell cycle profile analysis of the DNA histograms of integrated red fluorescence was performed using Modfit I 2.0 for Windows (Verity Software House, Inc., Topsham, ME, USA).

$R N A$ isolation and reverse transcription-quantitative polymerase chain reaction ( $R T-q P C R)$ analysis. Total RNA was isolated using the Ultrapure RNA kit (CwBio, Inc., Beijing, China). RNA quality was assessed by $1 \%$ agarose gel electrophoresis (AGE). The RNA was reverse transcribed using the HiFi-MMLV cDNA kit (CwBio-Electron, Inc.). The cDNA was subsequently amplified using UltraSYBR Mixture (with ROX; CwBio, Inc.). $\beta$-actin (ACTB) mRNA expression levels were used as an internal control to normalize the data. The primers were purchased from CwBio, Inc. (cat. no. CW0900). The Bmi-1 primers used in the present study were as follows: Bmi-1, forward 5'-TCCACCTCTTCTTGTTTGCCT-3', and reverse 5'-GAAGAAGTTGCTGATGACCCA-3'. All methods were conducted according to the manufacturer's instructions.

For miRNA-128a analysis, total RNA was isolated using the miRNApure Mini kit (CwBio, Inc.). RNA quality was assessed by AGE. The RNA was reverse transcribed using a miRNA cDNA kit (CwBio, Inc.). The cDNA was then amplified using a Real-Time PCR Assay kit (CwBio, Inc.). Small nuclear RNA U6 expression levels were used as an internal control to normalize the data. The primers were synthesized by $\mathrm{CwBio}$, Inc. The sequences of the primers used were as follows: U6, forward 5'-GCTTCGGCAGCACATATACTAAAAT-3'; and miRNA-128a, forward 5'-CACAGTGAACCGGTCTCTTT-3'.

The mRNA and miRNA expression levels were measured by RT-qPCR using an LC-480II thermal cycler (Roche Diagnostics, Basel, Switzerland). The cycling conditions were as follows: $95^{\circ} \mathrm{C}$ for $10 \mathrm{~min}$, followed by 40 cycles of amplification at $95^{\circ} \mathrm{C}$ for $15 \mathrm{sec}$ and $60^{\circ} \mathrm{C}$ for $60 \mathrm{sec}$. The relative miRNA and mRNA expression levels were calculated using the $\Delta \Delta C T$ method (20).

Analysis of the levels of intracellular reactive oxygen species $(R O S)$. The fluorescent probe 2',7'-dichlorodihydrofluorescein 
diacetate (DCFH-DA; Sigma-Aldrich) is able to pass through the cytomembrane, and is cleaved by nonspecific intracellular esterases in order to form 2',7'-dichlorodihydrofluorescein. DCFH-DA is also oxidized by ROS to form highly fluorescent 2',7'-dichlorofluorescein (DCF). The fluorescence intensity of DCF is therefore proportional to the levels of ROS in the cells. The U-87 MG glioma cells were exposed to various doses of X-ray radiation. After $6 \mathrm{~h}$, the cells were digested by $0.25 \%$ trypsin (Sigma-Aldrich) and harvested. The cells were washed twice with PBS, and incubated with $10 \mu \mathrm{M}$ DCFH-DA for $20 \mathrm{~min}$ in the dark at $37^{\circ} \mathrm{C}$. During incubation, the cells were gently agitated every 3-5 min. The cells were then washed three times with MEM in the absence of FBS, prior to being resuspended in $1 \mathrm{ml}$ PBS. The levels of ROS were assessed by FCM using an LSR Fortessa cell analyzer (BD Biosciences) at an excitation wavelength of $488 \mathrm{~nm}$ and emission wavelength of $530 \mathrm{~nm}$. The DCF fluorescence intensity data were evaluated by CellQuest-Pro 5.1 ${ }^{\mathrm{TM}}$ (BD Biosciences) and presented as the mean fluorescence intensity.

NAC treatment. The U87-MG cells were divided into control group, 8 Gy group and 8 Gy group with NAC treatment. Prior to $1 \mathrm{~h}$ exposure to an $8 \mathrm{~Gy}$ dose of $\mathrm{X}$-ray radiation, the culture medium in the groups subjected to NAC treatment was replaced with the MEM supplemented with $10 \%$ FBS and $1 \mathrm{mM}$ NAC. The culture medium in the control group and 8 Gy group were changed with MEM with $10 \%$ FBS (without NAC). All groups were incubated until being exposed to radiation, Following a $12 \mathrm{~h}$ exposure to radiation, the medium in all groups was replaced by MEM supplemented with $10 \%$ FBS.

Statistical analysis. Each experiment was conducted in triplicate. The results were presented as the mean \pm standard error. Student's t-test (unpaired) was used to evaluate the statistically significant differences. Statistical analysis was performed using SPSS version 13.0 (SPSS, Inc., Chicago, IL, USA). $\mathrm{P}<0.05$ was considered to indicate a statistically significant difference.

\section{Results}

Growth of U-87 MG glioma cells following X-ray exposure. In our previous study, the number of U-87 MG glioma cells did not decrease following exposure to various doses of X-ray radiation (17). To investigate whether various X-ray radiation fractions affected cellular growth, the U-87 MG cells were divided into three experimental groups: A conventional group, a hypofraction group, and a superfraction radiation group, which were exposed to X-ray radiation at 2 Gy qd for 5 days, 1.1 bid Gy for 5 days and 8 Gy for 1 day, respectively (Fig. 1). Cell growth was significantly inhibited in the hypofraction radiation group. Cell growth of the conventional and superfraction radiation groups was not inhibited until day 5, and no significant difference was observed between the two groups. Cell numbers increased in a time-dependent manner in all radiation groups, which suggests that the U-87 MG glioma cells exhibit radioresistance.

Inhibition ratio in $U-87$ MG glioma cells following $X$-ray exposure. To further investigate the inhibitory effects of X-ray

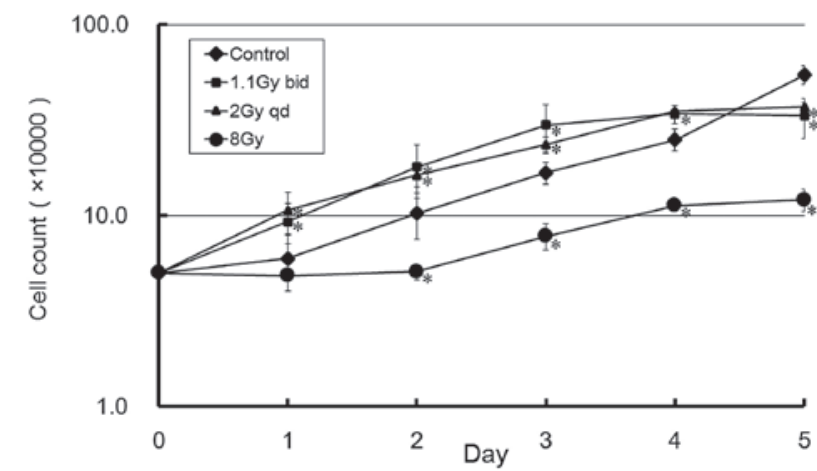

Figure 1. Cell growth curves of U87 MG glioma cells exposed to various doses of X-ray radiation.

radiation on the $\mathrm{U}-87 \mathrm{MG}$ cells, the cellular inhibition ratios were calculated following exposure to various radiation fractions (Fig. 2A) and various X-ray radiation doses (Fig. 2B). A significant increase in cell growth was detected in both the conventional and superfraction groups between days 1 and 4, and the same effects were observed in the 1 and 2 Gy groups. In the radiation groups exposed to $\geq 4 \mathrm{~Gy}$ radiation doses, the inhibitory effects on cellular growth were significant, and occurred in a dose-dependent manner. These results suggest that X-ray radiation is able to suppress the growth of $\mathrm{U}-87 \mathrm{MG}$ glioma cells. Our previous study demonstrated that the mechanism underlying radiation inhibition was cellular senescence, as opposed to apoptosis (17).

Cell cycle distribution of U-87 MG glioma cells following $X$-ray exposure. Our previous study investigated the cell cycle distribution of U-87 MG cells following $72 \mathrm{~h}$ exposure to $\mathrm{X}$-ray radiation. The number of cells in $\mathrm{G}_{2} / \mathrm{M}$ phase was significantly increased, and the number of cells in $G_{0} / G_{1}$ phase were significantly decreased in the 6 and 8 Gy groups (17). Cell senescence is characterized by an irreversible cell cycle arrest in $\mathrm{G}_{0} / \mathrm{G}_{1}$ phase. In our previous study, in the 6 and $8 \mathrm{~Gy}$ groups following $72 \mathrm{~h}$ exposure to radiation, the number of senescent cells was markedly increased; however, the number of cells in $\mathrm{G}_{0} / \mathrm{G}_{1}$ phase was markedly decreased (17). These results suggest that U-87 MG glioma cells exhibit cellular senescence evasion. To investigate whether the evasion of senescence occurred at earlier time points, the cell cycle distribution of the U-87 MG cells was analyzed following a $24 \mathrm{~h}$ exposure to $\mathrm{X}$-ray radiation (Fig. 3). The results indicate that the proportion of cells in S phase was significantly increased in the 1 and 2 Gy groups, and significantly decreased in the 6 and 8 Gy groups $(P<0.05)$. The proportion of cells in $\mathrm{G}_{2} / \mathrm{M}$ phase was significantly decreased in all radiation groups. The proportion of cells in $\mathrm{G}_{0} / \mathrm{G}_{1}$ phase increased in a dose-dependent manner. These results suggest that evasion of senescence may not occur following $24 \mathrm{~h}$ exposure to X-ray radiation.

mRNA expression levels of Bmi-1 in U-87 MG glioma cells following exposure to $X$-ray radiation. Our previous study determined that the protein expression levels of Bmi-1 were significantly increased in the 6 and 8 Gy groups following $72 \mathrm{~h}$ exposure to X-ray radiation (17), which suggested that an increase in Bmi-1 expression may underlie evasion of 

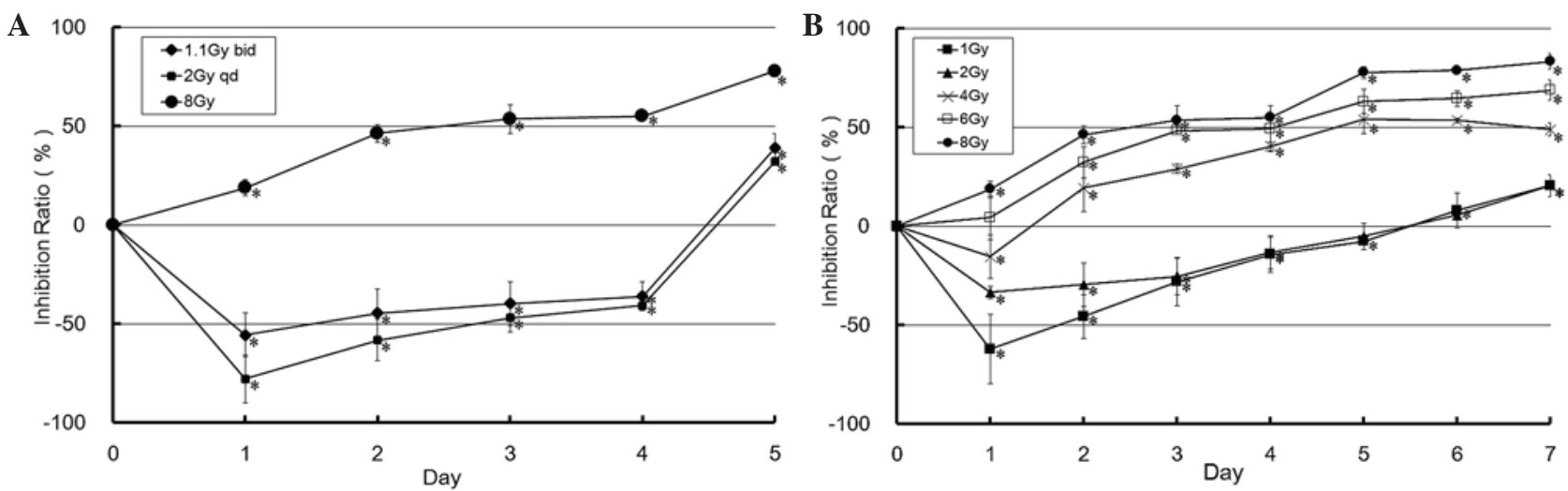

Figure 2. Inhibition ratio of the U-87 MG glioma cells exposed to X-ray radiation. The cells were exposed to (A) various radiation fractions or (B) various doses of X-ray radiation. Every $24 \mathrm{~h}$ the number of cells in three random wells were counted using a cell counter, and the mean value of the inhibition ratio, as compared with the control group was calculated.

A
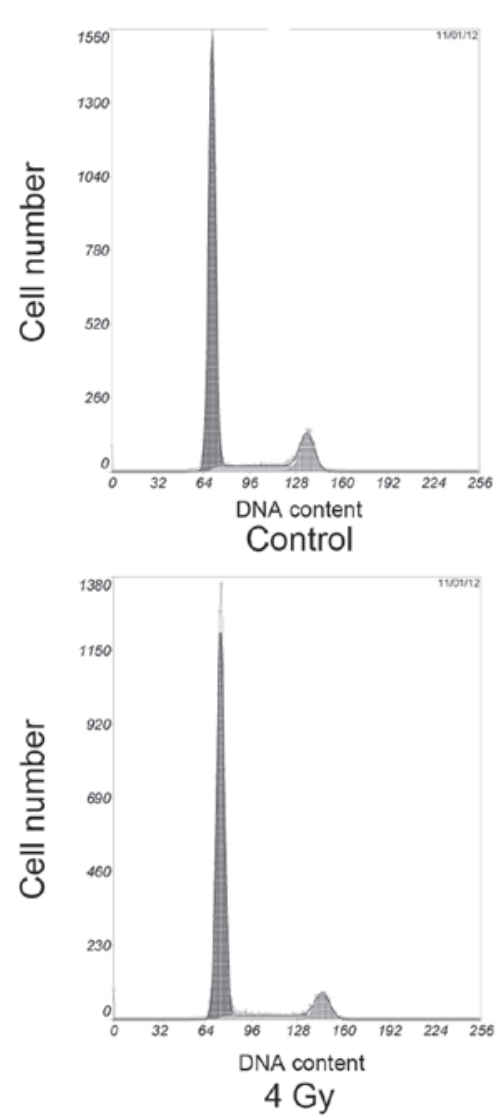
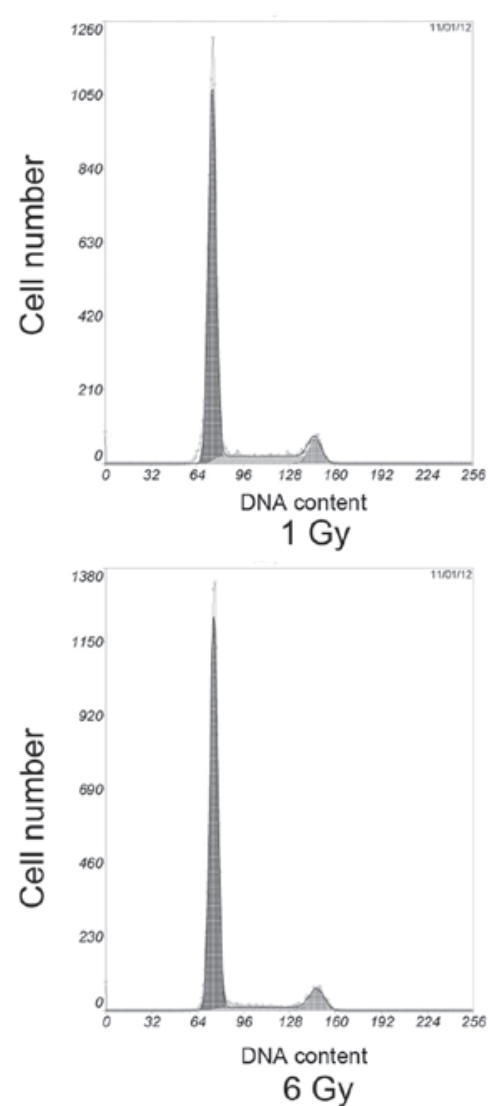
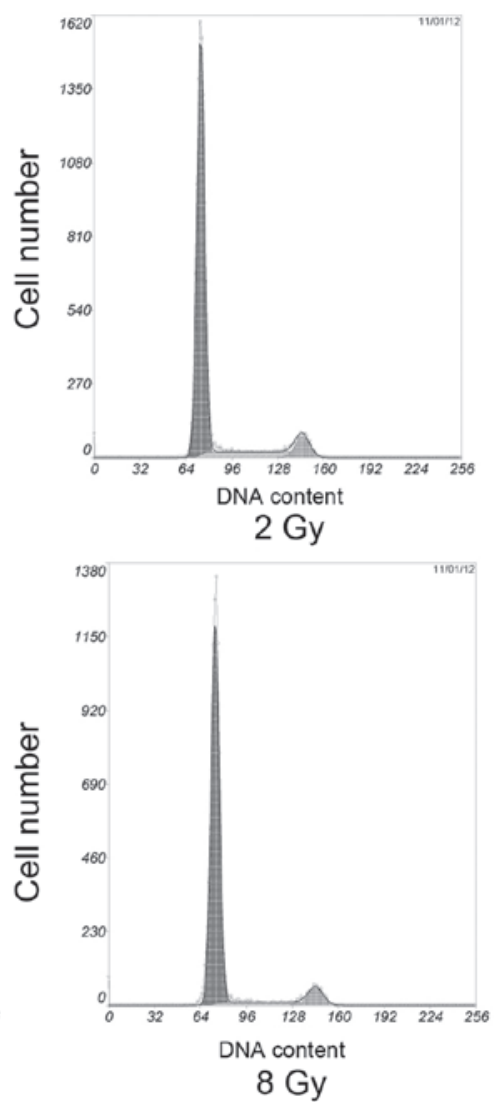

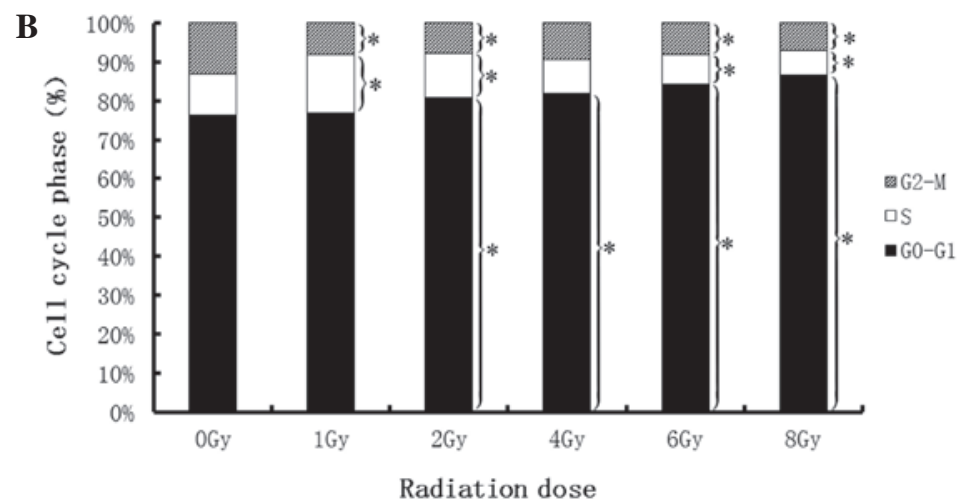

Figure 3. Cell cycle profiles of the U87 MG glioma cells. (A) Following $24 \mathrm{~h}$ exposure to X-ray radiation at various doses, the U-87 MG glioma cells were stained with propidium iodide and analyzed in order to determine cell cycle distribution. (B) Cell cycle profiles of the U87 MG glioma cells following exposure to radiation. The means are representative of three independent experiments. 
A

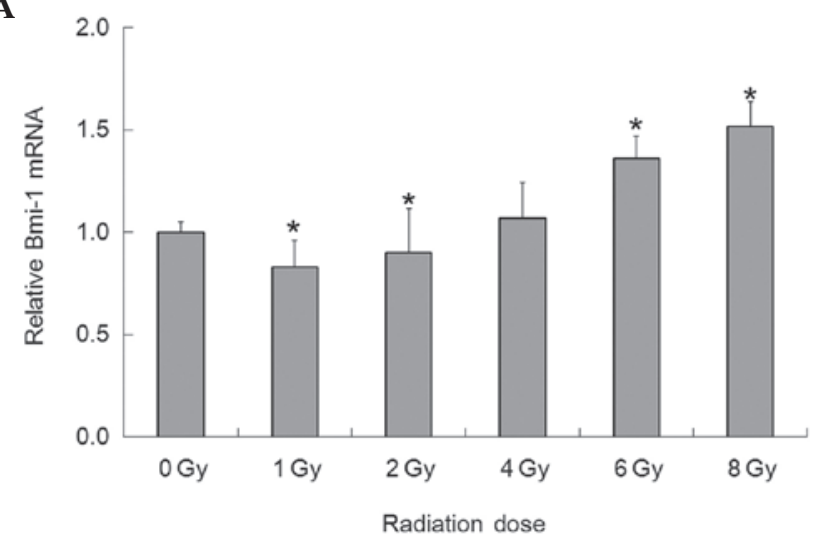

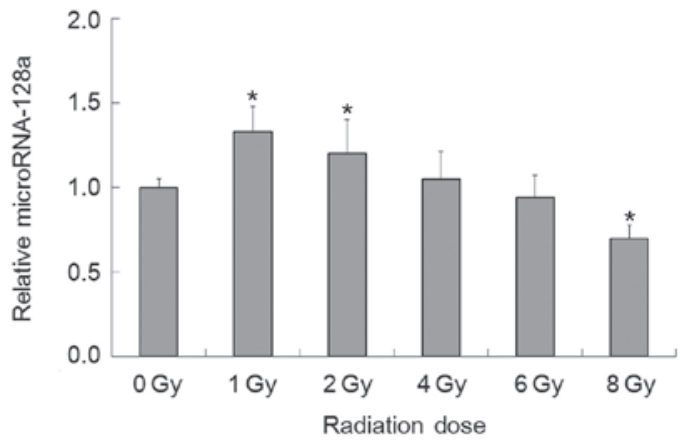

Figure 4. Relative expression levels of BMI1 polycomb ring finger oncogene (Bmi-1) mRNA and microRNA (miRNA)-128a following exposure to X-ray radiation. (A) mRNA expression levels of Bmi-1 and (B) expression levels of miRNA-128a in U-87 MG cells following $72 \mathrm{~h}$ exposure to various doses of $\mathrm{X}$-ray radiation, as determined by reverse transcription-quantitative polymerase chain reaction. The data are presented as the mean \pm standard error of three independent experiments. ${ }^{*} \mathrm{P}<0.05$, vs. the control group (0 Gy).
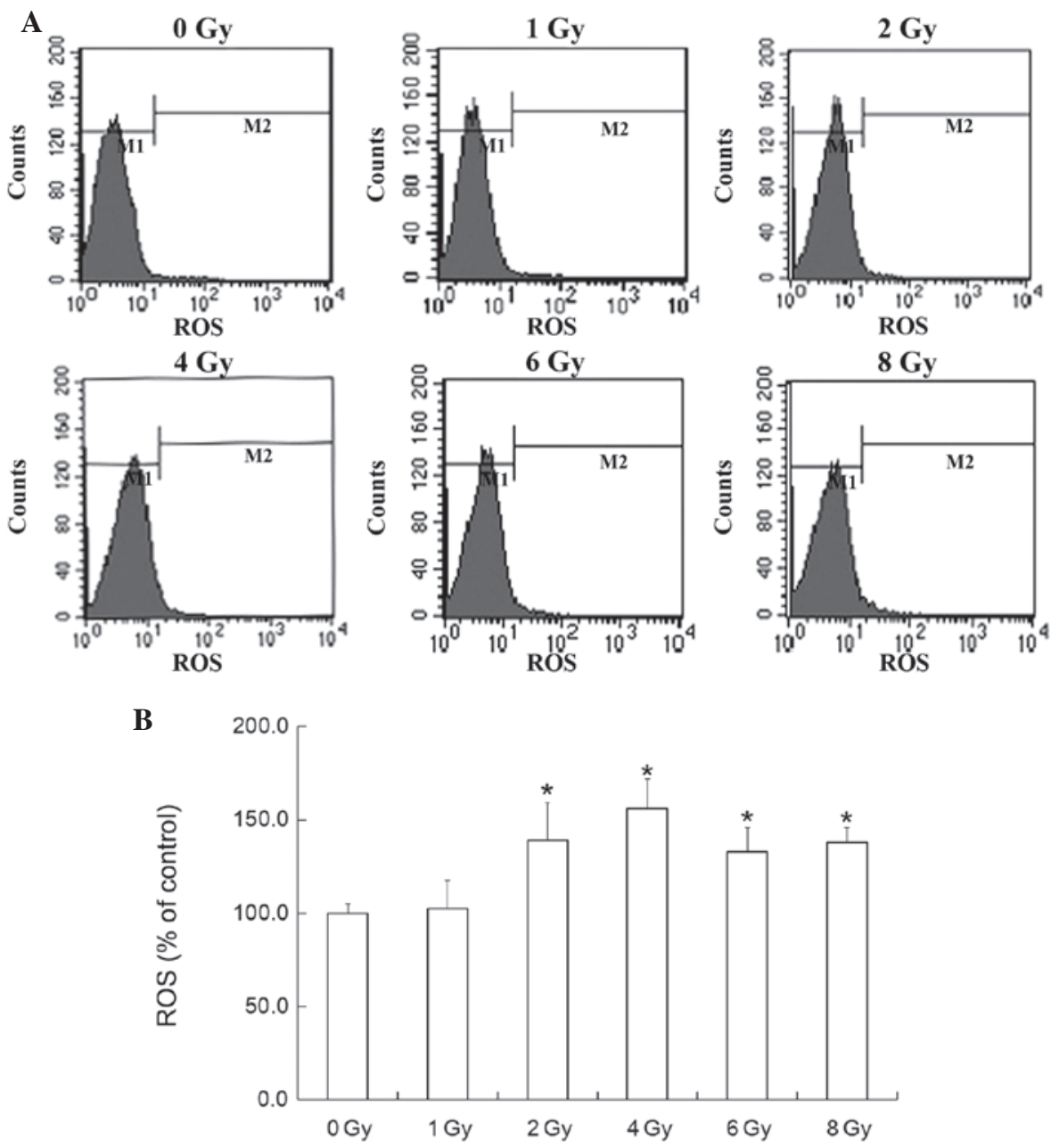

Radiation dose

Figure 5. (A) Intracellular reactive oxygen species (ROS) levels in the U-87 MG glioma cells following $6 \mathrm{~h}$ exposure to X-ray radiation, as detected by flow cytometry. (B) The ROS levels are presented as units of mean fluorescence intensity, and were calculated as a percentage of the control. ${ }^{*} \mathrm{P}<0.05$, vs. the control group.

senescence. To investigate whether the expression of Bmi-1 was affected by $\mathrm{X}$-ray radiation, the mRNA expression levels of Bmi-1 were detected in the U-87 MG cells following $72 \mathrm{~h}$ exposure to X-ray radiation by RT-qPCR (Fig. 4A). The mRNA expression levels of Bmi-1 were significantly decreased in the 1 and 2 Gy groups, and significantly increased in the 6 and 

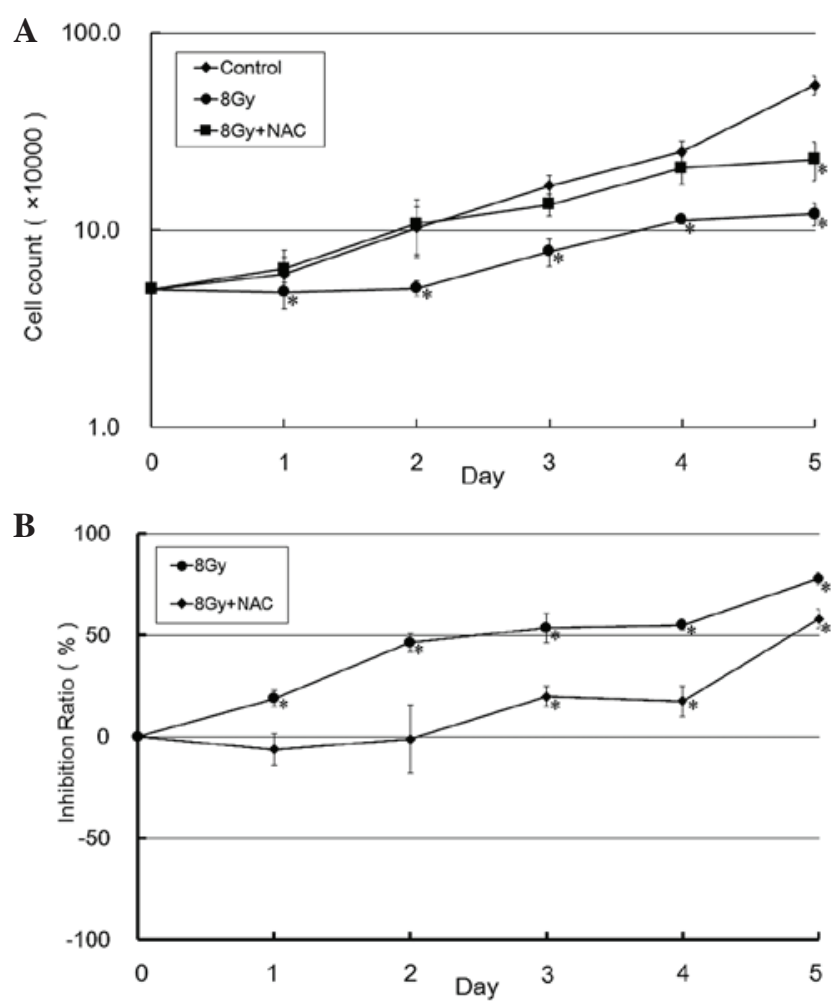

Figure 6. Effects of N-acetyl cysteine (NAC) on U-87 MG glioma cells following exposure to $\mathrm{X}$-ray radiation. Prior to $1 \mathrm{~h}$ exposure to $8 \mathrm{~Gy}$ dose $\mathrm{X}$-ray radiation, NAC was added to the minimal essential medium at a final concentration of $1 \mathrm{mM}$. (A) Cell growth curve and (B) inhibition ratio curve.

8 Gy groups. No significant difference was detected in the mRNA expression levels of Bmi-1 in the 4 Gy group.

miRNA-128a expression in U-87 MG glioma cells following exposure to $X$-ray radiation. To investigate the hypothesis that Bmi-1 expression is regulated by miRNA-128a, the expression levels of miRNA-128a were detected in the U-87 MG glioma cells by RT-qPCR, following $72 \mathrm{~h}$ exposure to X-ray radiation (Fig. 4B). The expression levels of miRNA-128a were significantly decreased in the 8Gy group, and increased in the 1 and 2 Gy groups. No significant difference was observed in the expression levels of miRNA-128a in the 4 and 6 Gy groups. These results indicate that the expression levels of miRNA-128a are negatively correlated with those of Bmi-1.

Effects of ROS on U-87 MG glioma cells following exposure to $X$-ray radiation. The intracellular levels of ROS in U-87 MG glioma cells were detected following $6 \mathrm{~h}$ exposure to X-ray radiation. The ROS levels in the 2, 4, 6 and 8 Gy groups were significantly increased, but no significant difference was identified in the 1 Gy group (Fig. 5).

$\mathrm{N}$-acetyl cysteine (NAC) is an active oxygen scavenger, which was used in the present study to investigate whether the levels of ROS were associated with the anti-tumorigenic effects of X-ray radiation. Prior to $1 \mathrm{~h}$ exposure to 8 Gy dose X-ray radiation, NAC was added to the MEM at a final concentration of $1 \mathrm{mM}$. The cell growth curve indicates that NAC is able to promote proliferation of U-87 MG glioma cells following exposure to $\mathrm{X}$-ray radiation, and the growth curve of the
NAC group was similar to that of the control group (Fig. 6A). The inhibition ratio of the NAC +8 Gy group was markedly decreased, as compared with the 8 Gy group (Fig. 6B). These results suggest that ROS has a significant role on the anti-tumorigenic effects of X-ray radiation, and reducing the levels of ROS may result in radioresistance.

\section{Discussion}

Glioma is the most prevalent type of primary (21) and malignant tumor (22) of the nervous system worldwide. Patients with glioblastoma grade IV have the shortest overall survival (23). Our previous study demonstrated that the U-87 MG human glioblastoma cell line is resistant to apoptosis and radiation (17). Evasion of cellular senescence may be the primary mechanism underlying the radioresistance of U-87 MG cells following exposure to X-ray radiation. In addition, Bmi-1 may have an important role in the radioresistance of $\mathrm{U}-87$ cells by promoting senescence evasion.

Radiotherapy is an important therapeutic strategy for the treatment of malignant tumors. Approximately 50\% of patients with malignant tumors require radiotherapy at a certain stage of their treatment, and this proportion increases to $92 \%$ in patients with nervous malignant tumors (24). The radioresistance of gliomas often decreases the efficacy of radiation (3); therefore, further research into increasing the radiosensitivity of gliomas may prove useful to prolong the overall survival time of patients with glioma.

Following exposure to various X-ray radiation fractions, the number of U-87 MG cells did not decrease in all experimental groups, which indicated that the U-87 MG glioma cells exhibited radioresistance. Analysis of inhibition ratios following exposure to X-ray radiation demonstrated a significant inhibition of U-87 MG glioma cell growth at an X-ray radiation dose $\geq 4 \mathrm{~Gy}$, indicating that the mechanism underlying cell growth inhibition was senescence, as opposed to apoptosis. At an X-ray dose $\leq 2 \mathrm{~Gy}$, the growth of U-87 MG cells was markedly increased in the initial few days, and after 5 days inhibition of growth occurred in response to both single and constant doses of radiation. In clinical settings, $\mathrm{X}$-ray radiation doses $\leq 2 \mathrm{~Gy}$ are usually used to avoid normal brain tissue injury. Therefore, patients with glioma, specifically glioblastoma, may safely undergo constant radiation for five days.

Senescence is an irreversible cell cycle arrest characterized by anti-apoptotic gene expression (25). It is widely accepted that tumor cells are able to evade senescence in order to maintain their proliferative ability. Therefore, research into the stimulation of cellular senescence in tumor cells merits further study (26). The cell cycle usually arrests permanently at $G_{0} / G_{1}$ phase during senescence. The results of the present study indicated that the proportion of cells in $\mathrm{G}_{2} / \mathrm{M}$ phase decreased significantly in all radiation groups, and the proportion of cells in $G_{0} / G_{1}$ phase increased significantly in a dose-dependent manner, following $24 \mathrm{~h}$ exposure to X-ray radiation. These results were concordant with the changes observed in the cell cycle during senescence. The results of the present study determined that senescence evasion may not occur following $24 \mathrm{~h}$ exposure to X-ray radiation. However, following $72 \mathrm{~h}$ exposure to radiation, the proportion of cells in 
the $G_{0} / G_{1}$ phase was significantly decreased in the 6 and 8 Gy groups, whereas the protein expression levels of Bmi-1 were significantly increased (17). The occurrence of senescence may induce Bmi-1 expression resulting in senescence evasion.

Bmi-1 belongs to the polycomb group gene family, which regulates cellular proliferation. Bmi-1 acts on the Ink4a-Arf genetic locus, resulting in the downregulation of p16Ink4a and p19Arf (p14Arf in human) (27). The $\mathrm{P} 16 /$ retinoblastoma $(\mathrm{Rb}) / \mathrm{E} 2 \mathrm{~F}$ signaling pathway has an important role in tumor development, and promotes glioma progression. Bmi-1 is able to suppress p16Ink4a, thereby increasing the activity levels of E2F1 (28). P16Ink4a is a specific inhibitor of cyclin-dependent kinase (CDK), which inhibits $\mathrm{Rb}$ via phosphorylation. $\mathrm{CDK}$ regulates passage from the $\mathrm{G}$ phase to the $\mathrm{S}$ phase of the cell cycle. A previous study demonstrated that when $\mathrm{Rb}$ expression is downregulated, the protein expression levels of E2F significantly increased resulting in abnormal cell proliferation (29). P16Ink4a expression is an indicator of cellular senescence (30). The P19Arf/mouse double minute 2 homolog (MDM2)/p53 is another significant signaling pathway responsible for tumor suppression. P19Arf is able to stabilize p53 by suppressing MDM2, and activating p53-dependent transcription, as a result, the cell cycle may be arrested in $G_{1}$ or $G_{2} / M$ phases prior to apoptosis (31). Due to the fact that U-87 MG glioma cells did not undergo apoptosis following exposure to $\mathrm{X}$-ray radiation, Bmi-1-induced senescence evasion may act via the $\mathrm{P} 16 / \mathrm{Rb} / \mathrm{E} 2 \mathrm{~F}$ signaling pathway, as opposed to the P19Arf/MDM2/P53 signaling pathway. Our previous study demonstrated that the expression levels of Bmi-1 were significantly increased following exposure to $\mathrm{X}$-ray radiation in the 6 and 8 Gy groups (17), and the present study demonstrated that the mRNA expression levels of Bmi-1 also significantly increased, which indicated that the expression levels of Bmi-1 may be increased at the transcription level, as opposed to the translation level. Therefore, senescence evasion may be suppressed via the inhibition of Bmi-1 gene transcription. However, the mechanism underlying the upregulation of Bmi-1 expression in U-87 MG glioma cells following $\geq 6$ Gy dose $\mathrm{X}$-ray radiation remains to be fully elucidated.

miRNA-128a is able to suppress Bmi-1 expression. To investigate whether miRNA-128a was involved in the response of glioma cells following exposure to X-ray radiation by targeting Bmi-1, the expression levels of miRNA-128a were evaluated by RT-qPCR. The results demonstrated that miRNA-128a expression was affected by X-ray radiation. The expression levels of miRNA-128a were significantly increased in the 1 and 2 Gy groups, and significantly decreased in the 8 Gy group. No significant difference was observed in the expression levels of miRNA-128a between the 4 and 6 Gy groups, and the control group; however, miRNA-128a expression was suppressed by X-ray radiation in a dose-dependent manner. Thus suggesting that miRNA-128a may participate in the regulation of Bmi-1 expression in U-87 MG cells following exposure to $\mathrm{X}$-ray radiation.

miRNA-128a was able to regulate the intracellular levels of ROS by targeting Bmi-1, thereby promoting senescence and inhibiting the growth of medulloblastoma cells (32). ROS are able to modulate various cellular functions, including tumor cell biology (33). Radiation stimulates the generation of
ROS in tumor cells resulting in apoptosis, senescence, or cell death (34). The present study demonstrated that ROS levels in the U-87 MG cells were significantly increased following $\geq 2$ Gy dose X-ray radiation. Following treatment with the ROS scavenger NAC, the growth of the U-87 MG cells was markedly increased, and the inhibitory effects of X-ray radiation were markedly decreased. Therefore, the decrease in the levels of ROS may induce radioresistance in U-87MG glioma cells. One of the possible mechanisms underlying the senescence evasion of U-87MG glioma cells is a decrease in miRNA-128a expression following exposure to $\mathrm{X}$-ray radiation, which results in Bmi-1 gene transcription upregulation. This would then lead to reduced levels of intracellular ROS, resulting in senescence evasion.

The present study demonstrated the radioresistance of U-87 MG cells. Exposure to radiation was able to increase the mRNA expression levels of Bmi-1 resulting in senescence evasion, which may function via the $\mathrm{p} 16 / \mathrm{Rb} / \mathrm{E} 2 \mathrm{~F}$ signaling pathway. miRNA-128a and its downstream gene Bmi-1 may be important for the radioresistance of U-87 MG glioma cells. In addition, ROS may be important for the inhibitory effects induced by X-ray radiation in U-87 MG cells. However, the present study had the following limitations: (i) The study investigated U-87 MG glioma cells only, and therefore the conjectures obtained from the results may not be significant for other types of gliomas; (ii) the mechanism underlying the downregulated generation of ROS, which resulted in radioresistance remains to be elucidated; (iii) no conclusive association was observed between ROS levels and the expression levels of Bmi-1 and miRNA-128a; and (iv) although the expression levels of Bmi-1 and miRNA-128a were altered in the U-87 MG cells following exposure to X-ray radiation, whether radiosensitivity is increased if the expression levels of Bmi-1 or miRNA-128a are downregulated remains to be determined. Further research is required in order to elucidate these mechanisms.

\section{Acknowledgements}

The present study was supported by the Youth Foundation in the Second Hospital of Shandong University (grant no. 2013010016), the Jinan University Institute Independent Innovation Plan (grant no. 201401263) and the Shandong Provincial Natural Science Foundation (grant no. R2013HL027).

\section{References}

1. Haris K, Ismail S, Idris Z, Abdullah JM and Yusoff AA: Expression profile of genes modulated by Aloe emodin in human U87 glioblastoma cells. Asian Pac J Cancer Prev 15: 4499-4505, 2014 .

2. Lee JH, Jung TY, Jung S, Kim IY, Jang WY, Moon KS and Jeong EH: Performance status during and after radiotherapy plus concomitant and adjuvant temozolomide in elderly patients with glioblastoma multiforme. J Clin Neurosci 20: 503-508, 2013.

3. Stupp R, Hegi ME, Mason WP, van den Bent MJ, Taphoorn MJ, Janzer RC, Ludwin SK, Allgeier A, Fisher B, Belanger K, et al; European Organisation for Research and Treatment of Cancer Brain Tumour and Radiation Oncology Groups; National Cancer Institute of Canada Clinical Trials Group: Effects of radiotherapy with concomitant and adjuvant temozolomide versus radiotherapy alone on survival in glioblastoma in a randomised phase III study: 5-year analysis of the EORTC-NCIC trial. Lancet Oncol 10: 459-466, 2009. 
4. Wick W, Weller M, van den Bent M, Sanson M, Weiler M, von Deimling A, Plass C, Hegi M, Platten M and Reifenberger G: MGMT testing - the challenges for biomarker-based glioma treatment. Nat Rev Neurol 10: 372-385, 2014.

5. Errafiy R, Aguado C, Ghislat G, Esteve JM, Gil A, Loutfi M and Knecht E: PTEN increases autophagy and inhibits the ubiquitin-proteasome pathway in glioma cells independently of its lipid phosphatase activity. PLoS One 8: e83318, 2013.

6. Lim YC, Roberts TL, Day BW, Harding A, Kozlov S, Kijas AW, Ensbey KS, Walker DG and Lavin MF: A role for homologous recombination and abnormal cell-cycle progression in radioresistance of glioma-initiating cells. Mol Cancer Ther 11: 1863-1872, 2012.

7. Ketting RF: microRNA biogenesis and function : An overview. Adv Exp Med Biol 700: 1-14, 2011.

8. Ambros V: The functions of animal microRNAs. Nature 431: $350-355,2004$

9. Giraldez AJ, Cinalli RM, Glasner ME, Enright AJ, Thomson JM, Baskerville S, Hammond SM, Bartel DP and Schier AF: MicroRNAs regulate brain morphogenesis in zebrafish. Science 308: 833-838, 2005.

10. Lu J, Getz G, Miska EA, Alvarez-Saavedra E, Lamb J, Peck D, Sweet-Cordero A, Ebert BL, Mak RH, Ferrando AA, et al: MicroRNA expression profiles classify human cancers. Nature 435: 834-838, 2005

11. Png KJ, Halberg N, Yoshida M and Tavazoie SF: A microRNA regulon that mediates endothelial recruitment and metastasis by cancer cells. Nature 481: 190-194, 2012.

12. Macfarlane LA and Murphy PR: MicroRNA: Biogenesis, function and role in cancer. Curr Genomics 11: 537-561, 2010.

13. Kumar MS, Lu J, Mercer KL, Golub TR and Jacks T: Impaired microRNA processing enhances cellular transformation and tumorigenesis. Nat Genet 39: 673-677, 2007.

14. Corsten MF, Miranda R, Kasmieh R, Krichevsky AM, Weissleder R and Shah K: MicroRNA-21 knockdown disrupts glioma growth in vivo and displays synergistic cytotoxicity with neural precursor cell delivered S-TRAIL in human gliomas. Cancer Res 67: 8994-9000, 2007.

15. Cui QK, Liu WD, Zhu JX, Wang YH and Wang ZG: MicroRNA-184 promotes proliferation ability of glioma cells by regulating FOXO3. Asian Pac J Trop Med 7: 776-779, 2014.

16. Hu X, Chen D, Cui Y, Li Z and Huang J: Targeting microRNA-23a to inhibit glioma cell invasion via HOXD10. Sci Rep 3: 3423, 2013.

17. Ye L, Wang C, Yu G, Jiang Y, Sun D, Zhang Z, Yu X, Li X, Wei W, Liu P, et al: Bmi-1 induces radioresistance by suppressing senescence in human U87 glioma cells. Oncol Lett 8: 2601-2606, 2014.

18. Ciafrè SA, Galardi S, Mangiola A, Ferracin M, Liu CG, Sabatino G, Negrini M, Maira G, Croce CM and Farace MG: Extensive modulation of a set of microRNAs in primary glioblastoma. Biochem Biophys Res Commun 334: 1351-1358, 2005
19. Godlewski J, Nowicki MO, Bronisz A, Williams S, Otsuki A, Nuovo G, Raychaudhury A, Newton HB, Chiocca EA and Lawler S: Targeting of the Bmi-1 oncogene/stem cell renewal factor by microRNA-128 inhibits glioma proliferation and self-renewal. Cancer Res 68: 9125-9130, 2008.

20. Livak KJ and Schmittgen TD: Analysis of relative gene expression data using real-time quantitative PCR and the 2(-Delta Delta C(T)) Method. Methods 25: 402-408, 2001

21. Murayama S, Kawai R, Hirabuki N, Miura T, Mitomo M, Kozuka T and Usio Y: Intra-arterial ACNU chemotherapy of malignant glioma. Nihon Igaku Hoshasen Gakkai Zasshi 48: 144-153, 1988 (In Japanese).

22. Taylor LP: Diagnosis, treatment, and prognosis of glioma: Five new things. Neurology (18 Suppl 1) 75: S28-S32, 2010

23. Clarke J, Butowski $N$ and Chang S: Recent advances in therapy for glioblastoma. Arch Neurol 67: 279-283, 2010.

24. Delaney G, Jacob S, Featherstone $C$ and Barton M: The role of radiotherapy in cancer treatment: Estimating optimal utilization from a review of evidence-based clinical guidelines. Cancer 104: 1129-1137, 2005

25. Schmitt CA: Cellular senescence and cancer treatment. Biochim Biophys Acta 1775: 5-20, 2007.

26. Roninson IB: Tumor cell senescence in cancer treatment. Cancer Res 63: 2705-2715, 2003.

27. Molofsky AV, He S, Bydon M, Morrison SJ and Pardal R: Bmi-1 promotes neural stem cell self-renewal and neural development but not mouse growth and survival by repressing the p16Ink4a and p19Arf senescence pathways. Genes Dev 19: 1432-1437, 2005.

28. O'Donnell KA, Wentzel EA, Zeller KI, Dang CV and Mendell JT: c-Myc-regulated microRNAs modulate E2F1 expression. Nature 435: 839-843, 2005.

29. Maher EA, Furnari FB, Bachoo RM, Rowitch DH, Louis DN, Cavenee WK and DePinho RA: Malignant glioma: Genetics and biology of a grave matter. Genes Dev 15: 1311-1333, 2001.

30. Hara E, Smith R, Parry D, Tahara H, Stone S and Peters G: Regulation of p16CDKN2 expression and its implications for cell immortalization and senescence. Mol Cell Biol 16: 859-867, 1996.

31. Zhang Y, Xiong Y and Yarbrough WG: ARF promotes MDM2 degradation and stabilizes p53: ARF-INK4a locus deletion impairs both the $\mathrm{Rb}$ and p53 tumor suppression pathways. Cell 92: 725-734, 1998.

32. Venkataraman S, Alimova I, Fan R, Harris P, Foreman N and Vibhakar R: MicroRNA 128a increases intracellular ROS level by targeting Bmi-1 and inhibits medulloblastoma cancer cell growth by promoting senescence. PLoS One 5: e10748, 2010.

33. Finkel T: Signal transduction by reactive oxygen species in non-phagocytic cells. J Leukoc Biol 65: 337-340, 1999.

34. Bauer G: Low dose radiation and intercellular induction of apoptosis: Potential implications for the control of oncogenesis. Int J Radiat Biol 83: 873-888, 2007. 